\title{
THE USE OF VACUUM PACKED PARTICLES WITH ADAPTABLE PROPERTIES IN ACOUSTIC APPLICATIONS
}

\author{
Robert Zalewski, Michą Rutkowski \\ Warsaw University of Technology, Institute of Machine Design Fundamentals, Warsaw, Poland \\ e-mail: landranger@gmail.com; robertzalewski@wp.pl
}

\begin{abstract}
The aim of this paper is to present possibilities of using a new type of granular media in acoustics as innovative sound absorbers. Most materials of this type have a porous or fibrous structure. It is constant, and once manufactured, does not easily change its configuration. The examined material - Vacuum Packed Particles (VPP) is of a changeable structure. It can be assumed that the acoustical absorption of such structures can be modified and partially adjusted by an external factor. First steps in an experimental approach have been made - the acquired results are optimistic. Additional tests are being planned to confirm the observed phenomenon and to apply VPP as novel materials in acoustics. Basing on the preliminary experimental tests, it can be concluded that the considered structures could become a significant part of a multilayered structure which would have controllable sound absorption properties.
\end{abstract}

Keywords: Vacuum Packed Particles, absorption coefficient, experiments, Voronina model

\section{List of abbreviations}

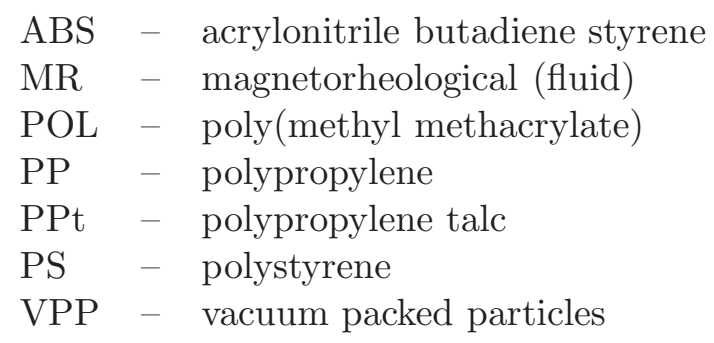

\section{Introduction}

Nowadays, there is demand for more efficient sound absorbing materials. It is obvious that all classical materials (porous, fibrous) are tested, and their acoustic characteristics could be found in many scientific publications. Most of the above are artificial and expensive structures (Karliński et al., 2014), and currently there is a general tendency to search for "green" and innovative solutions in the engineering. Many efforts have been made to increase the applicability of recyclable materials, production leftovers etc., as some recent publications demonstrate (Ersoy and Küçük, 2009; Hong et al., 2007; Fatima and Mohanty, 2011). Most frequently, the investigated sound absorbing materials show good absorption/transmission loss properties and, since most of acoustic tests are comprehensive (considering e.g. various thicknesses, densities, particle size, etc.), it is quite easy to determine general application possibilities and limitations concerning the researched materials (Gawdzińska et al., 2015, 2016).

The modeling of the behavior of various granular absorbing materials (mixes) in various states (compressed, loose) (Voronina and Horoshenkov, 2003; Swift et al., 1999; Wilson, 1999) 
has shown that although most of tested particles are made of widely known and well-tested materials, there are still possibilities of using them in a better and more effective way. Some general modifications to the porous materials structure are adding air gaps, combining few layers with different acoustic properties to create a novel sound absorber, adding an additional mass to the system or even active elements (Besset and Ichchou, 2011; Yamamoto et al., 2009; Zieliński, 2011). Following this path, the next step is an active absorbing material which would be able to adjust accordingly to a control measure, its own structure to better suit the requirements.

The presented research does not concern "green" materials directly, but the investigated materials could be treated as a typical leftover from production processes or products of recycling. Granulates used in the experiment were made of 5 different plastics. Using them as the so-called Vacuum Packed Particles consists in packing them in a thin hermetic envelope in which a partial vacuum (the so called underpressure) is generated.

Vacuum Packed Particles (VPP), with some limitations, can be treated as a type of a smart structure. When subjected to a partial vacuum, the structure greatly increases its apparent viscosity to the point of becoming a viscoplastic solid. Importantly, the yield stress of the VPP when in its active ("on") state can be controlled very accurately by varying the underpressure intensity (Bajkowski and Zalewski, 2014). The outcome of this is that the structure ability to transmit a force can be controlled with a vacuum pump, which increases the number of its possible control-based applications. Extensive discussions of the physics and applications of VPP fluids can be found in (Zalewski, 2013) or previous papers of the authors (Pyrz and Zalewski, 2010; Zalewski, 2010).

Controlling the vacuum range is controlling the structure properties, which is additionally a reversible process. As previously mentioned, VPP have some characteristics that are unique to smart materials. Moreover, their considerable advantage is the simplicity and a low price compared to other smart structures, e.g. MR fluids (Bajkowski et al., 2012) or (Zalewski et al., 2014), and common availability.

\section{Experimental set-up}

Acoustic properties have been tested according to an appropriate standard (ISO 10534-2) with the use of Brüel \& Kjær equipment: the impedance tube (4206) with two microphones (2670), (3160-A-04/2) generator and the (2716C) amplifier. The experimental setup is depicted in Fig. 1. Additional equipment consisted of a PC with Pulse Lab Shop software and a vacuum pump with two gauges for pressure control. The proposed experimental stand enabled obtaining reliable results between 500 and $6400 \mathrm{~Hz}$.

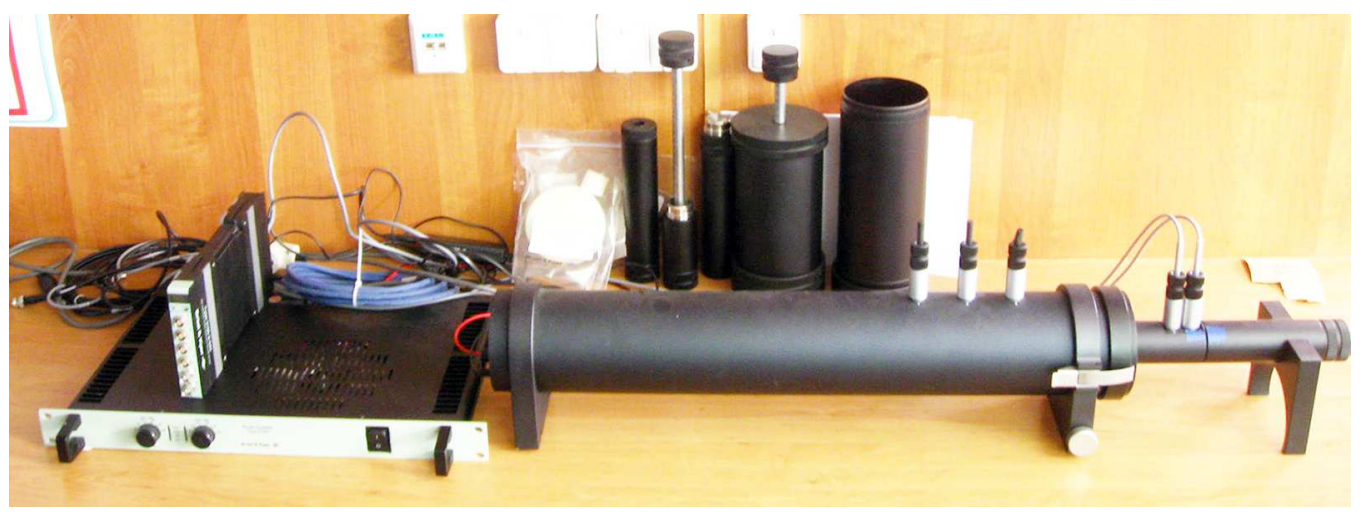

Fig. 1. The main part of the experimental setup 


\subsection{The range of experiments}

Granular structures have been investigated to determine the influence of several factors on the sound absorption coefficient. The following factors are particularly worthy of being mentioned:

- the level of underpressure generated inside the sample (ten various underpressure levels ranging from 0 to $0.09 \mathrm{MPa}$ with a step of $0.01 \mathrm{MPa}$ were taken into consideration),

- length of a sample (two different cylindrical specimens, i.e. sample I and II, having 130 and $200 \mathrm{~mm}$ in length, respectively, diameter of the specimen was constant and equal $D=29 \mathrm{~mm})$,

- the grains material (five various materials were considered: polypropylene (PP), polystyrene (PS), acrylonitrile butadiene styrene (ABS), poly(methyl methacrylate) (POL) and polypropylene talc (PPt)),

- the front surface of a specimen (three types of various thickness were tested: 0.05, 0.1 and, $0.2 \mathrm{~mm}$ for type 1, 2 and 3 , respectively).

To minimize measurement errors, the tests were performed in three independent experimental series. It was assumed that the internal porosity of investigated granular samples was constant and equal $N=0.28$. Dimensions of the considered barrel-roller shaped grains were $2-3 \mathrm{~mm}$ diameter and 4-5 mm length (Fig. 2). Densities of the tested materials are shown in Table 1 (values determined in separate laboratory tests).

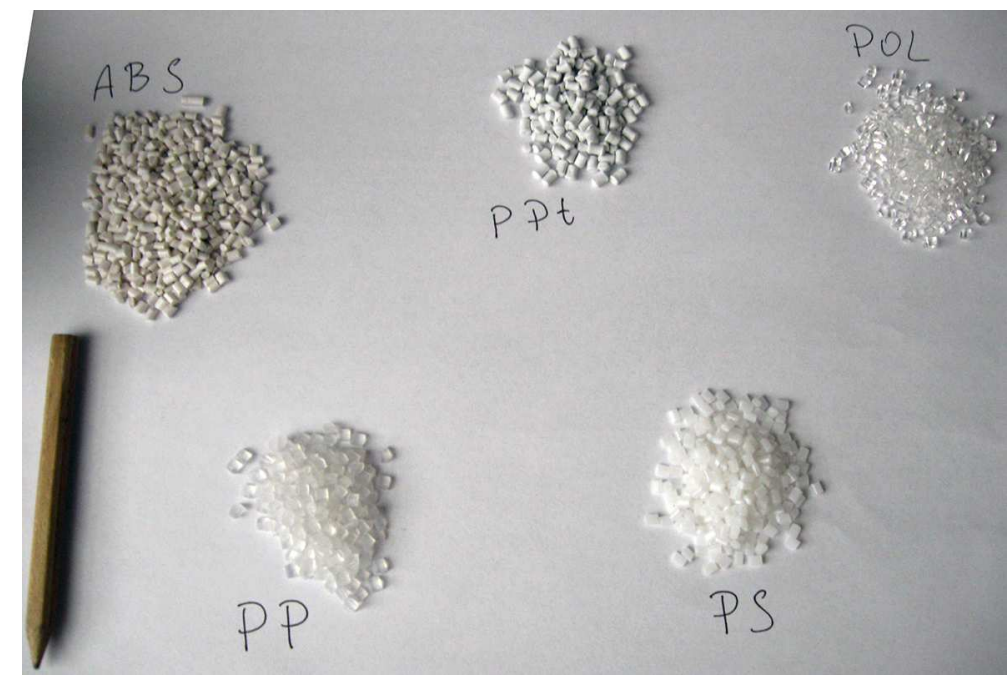

Fig. 2. Particles used in the specimen construction

Table 1. Densities of tested materials

\begin{tabular}{|c|c|}
\hline Grain material & Density $\left[\mathrm{g} / \mathrm{cm}^{3}\right]$ \\
\hline \hline $\mathrm{PP}$ & 0.92 \\
\hline $\mathrm{PS}$ & 1.06 \\
\hline $\mathrm{ABS}$ & 1.04 \\
\hline $\mathrm{POL}$ & 1.18 \\
\hline $\mathrm{PPt}$ & 1.21 \\
\hline
\end{tabular}

\subsection{Specimen construction}

The necessity to generate a partial vacuum inside the investigated testing pieces forced the authors to design a special construction of the experimental sample. The grains were packed in a 
thin plastic container as shown below in Fig. 3. The rear part of the sample was manufactured as a steel disc which acted as a "rigid" wall behind the test material. The sample previously mounted inside the impedance tube was in the next step connected to the vacuum pump. To verify such an approach to experimental research, a second type of specimen was designed, where the original parts of the mentioned impedance tube were used. Differences in the observed responses of the investigated materials were negligible.

Because the design of the test sample had to ensure tightness, its front was sealed by a thin plastic membrane (made of polypropylene, thicknesses $0.05-0.2 \mathrm{~mm}$ ). To try and find out the impact of the material in front of the sample, three various thickness values were analyzed (Section 2.1).

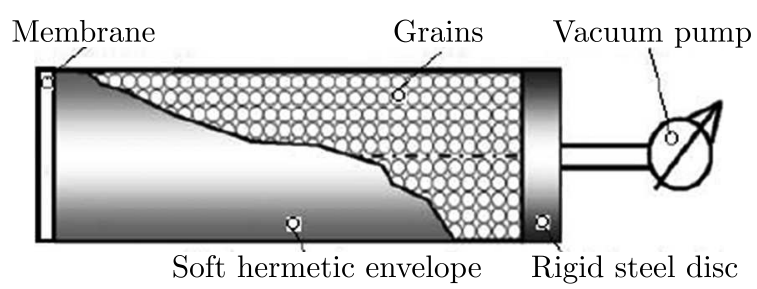

Fig. 3. Specimen construction details

\section{Results and discussion}

\subsection{Influence of the underpressure}

It can be observed in Figs. 4 and 5 (absorption coefficient of ABS and PP particles, respectively) that the underpressure generated inside the sample has a noticeable impact on acoustic properties of tested granular structures for the whole range of investigated frequencies. Moreover, the impact of a partial vacuum is nonlinear. The observed structural change, where the material is transforming from a semi-liquid to a semi-solid state (so called "jamming mechanism"), appears to be the strongest between the atmospheric pressure and $0.01 \mathrm{MPa}$ underpressure. Additional experimental tests conducted on other granular materials revealed a similar phenomenon, where the full phase transition was observed near the $0.03 \mathrm{MPa}$ underpressure limit. Beyond that threshold value, there is no evident difference in the recorded acoustic absorption variations. The changes in the granular structure are noticeable, since they affect every grain in the sample. Once submitted to underpressure, the grains are compacting. It results in a nonlinear increase in the number of contact points and simultaneously "intergranular" forces values (Pyrz and Zalewski, 2010). In this "jammed" state, the grains can no longer freely vibrate, also the air cavities surrounding them are greatly reduced or even eliminated (Brown et al., 2010).

\subsection{Influence of the front surface material}

Figures 6 and 7, also Table 2 show the influence of the front membrane of investigated samples filled with ABS and POL grains respectively on their acoustic absorption coefficient measured in the atmospheric pressure. The analyzed thicknesses were ranging approximately from $0.2 \mathrm{~mm}$ (type 3 ) to $0.05 \mathrm{~mm}$ (type 1). It is clearly depicted that acoustic properties of the samples under test are very poor for type 3 membrane. For type 2 they are significantly better and membrane type 1 is evidently the best in the whole range of experimental frequencies.

The differences between type 1 and 3 material are several hundred percent. Another observation can be made that for frequencies below $2-2.5 \mathrm{kHz}$, an increase in the absorbing efficiency (with decreasing thickness of the membrane) is considerably slower than for higher frequencies. Also, the membrane thickness seems to have a lot more impact on the absorption above $2 \mathrm{kHz}$. 


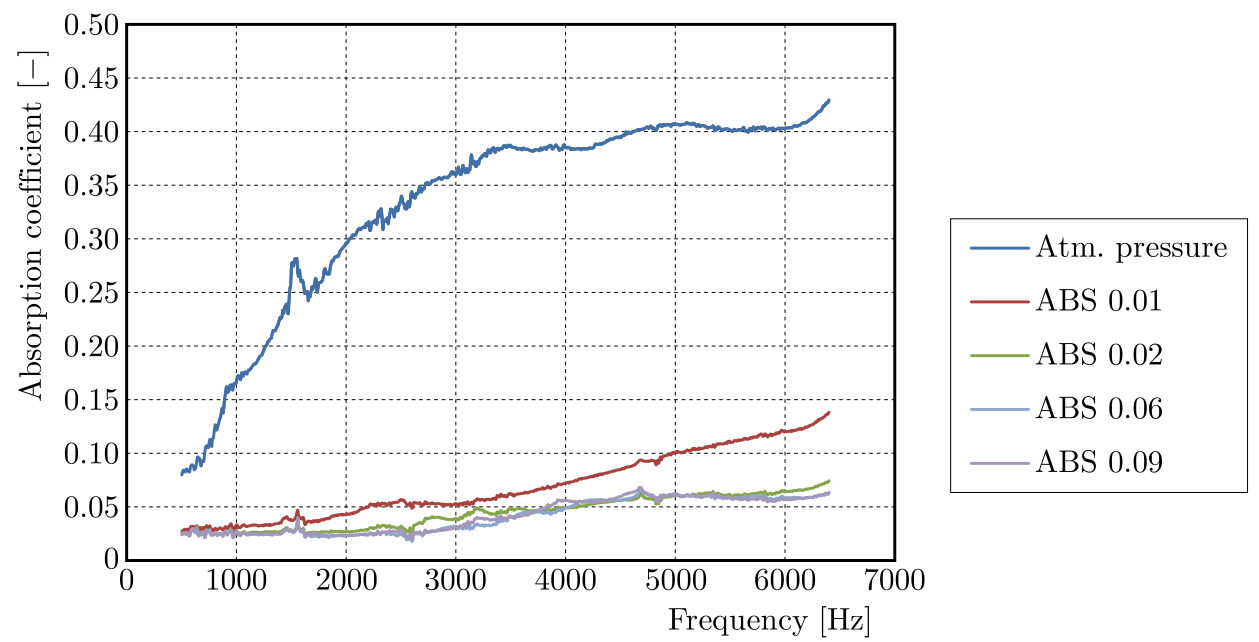

Fig. 4. Sound absorption coefficient for ABS grains and various underpressure values; sample II, front material type 2

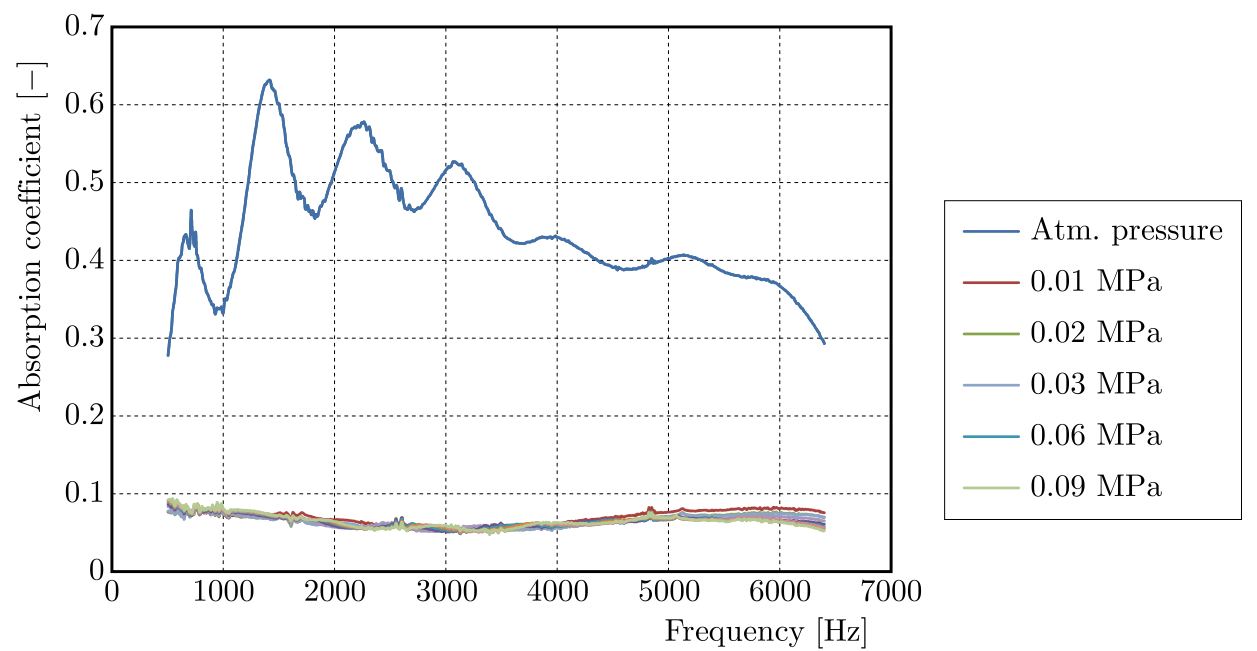

Fig. 5. Sound absorption coefficient for PP grains and various underpressure values; sample I, front material type 1

Table 2. Sound absorption coefficient of ABS and POL particles for various specimen front materials

\begin{tabular}{|c|c|c|c|c|c|c|}
\hline Grain & Sample front & \multicolumn{5}{|c|}{ Frequency [Hz] } \\
\cline { 3 - 7 } material & material type & 500 & 1000 & 2000 & 4000 & 6400 \\
\hline \hline \multirow{3}{*}{ ABS } & 3 & 0.057 & 0.052 & 0.058 & 0.059 & 0.088 \\
\cline { 2 - 7 } & 2 & 0.080 & 0.166 & 0.296 & 0.385 & 0.429 \\
\cline { 2 - 7 } & 1 & 0.267 & 0.448 & 0.429 & 0.547 & 0.464 \\
\hline \multirow{3}{*}{ POL } & 3 & 0.057 & 0.076 & 0.098 & 0.128 & 0.124 \\
\cline { 2 - 7 } & 2 & 0.126 & 0.239 & 0.390 & 0.482 & 0.407 \\
\cline { 2 - 7 } & 1 & 0.457 & 0.574 & 0.581 & 0.571 & 0.580 \\
\hline
\end{tabular}

The cause for some differences mentioned above would probably be an increase of sound waves penetration with a reduction in the membrane thickness. It is obvious that using a supplementary material in front of the tested grains simply limits their dissipation capabilities but, nevertheless, it is the only way to use Vacuum Packed Particles as controllable sound absorbers. 


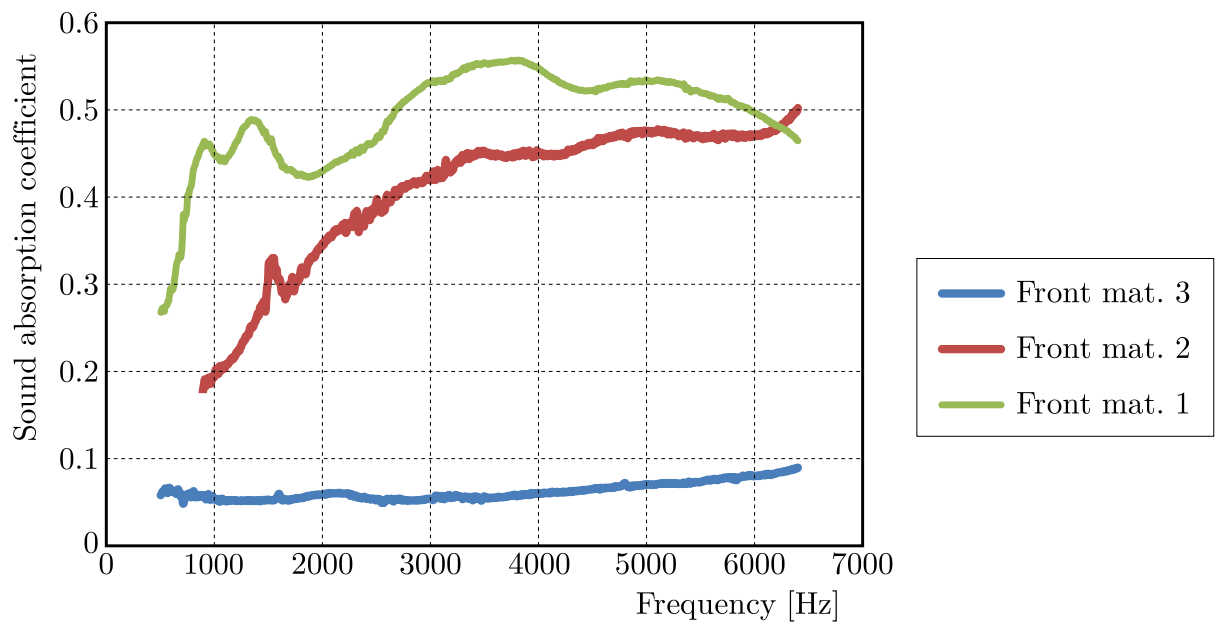

Fig. 6. Sound absorption coefficient for ABS grains subject to atmospheric pressure; sample I, various types of specimens front materials

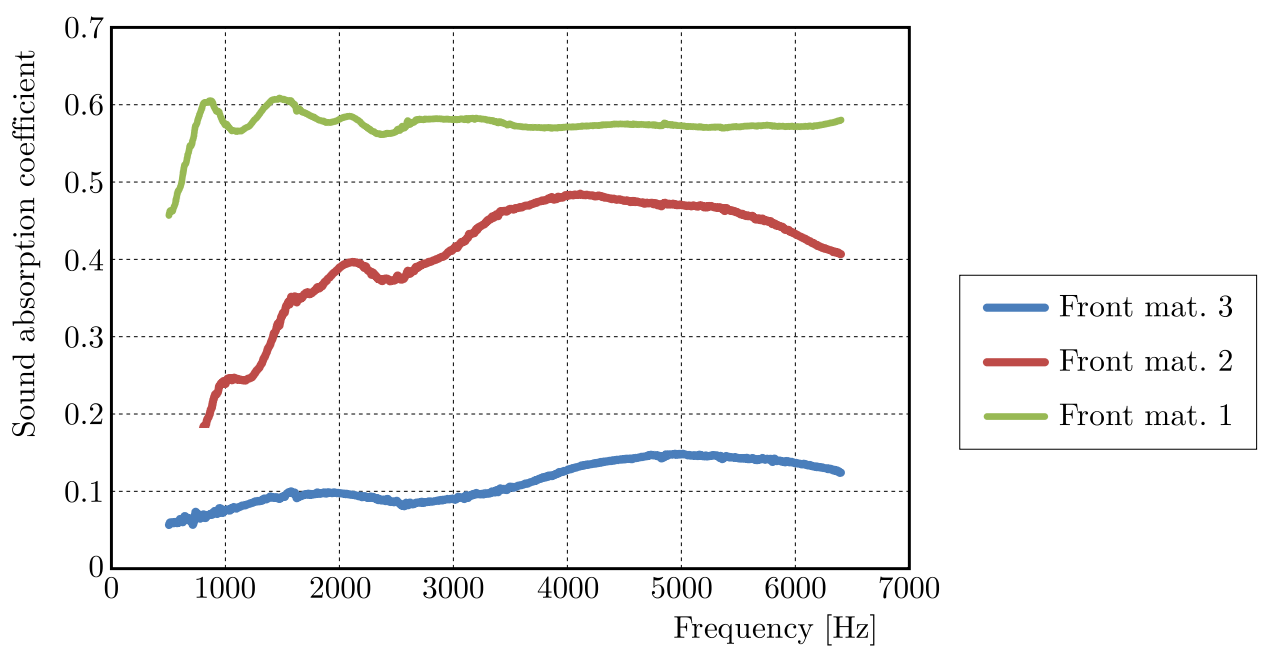

Fig. 7. Sound absorption coefficient for POL grains subject to atmospheric pressure; sample II, various types of specimens front materials

\subsection{Influence of the grain material}

In Fig. 8, various grains materials subjected to the atmospheric pressure are investigated and their sound absorption coefficient is depicted. It is additionally assumed that the front membrane is the same for all experiments analyzed in this Section (type 2). As for the shape of the curves, it can be stated that the characteristics of ABS and POL grains reveal many similarities, especially for the lower values of considered frequencies. Also in mid and high frequencies the value of coefficient seems to be close and stabilizing for both. The characteristics of PP and PS also seem to be close in shape with a clear highest value of the coefficient for both plastics. However, the highest value for both these materials is almost $1.5 \mathrm{kHz}$ apart in frequency. The highest sound absorption properties have been obtained for PS structures, for frequencies below $2.5 \mathrm{kHz}$, other materials showed best performance between 3 and $5 \mathrm{kHz}$. Such characteristics probably result from slight size/shape variations in the grain mixes as well as the materials density scatter. A common feature for all 4 curves is a similar peak that can be found at about $1.5 \mathrm{kHz}$ as well as the characteristic below the value of $1.5 \mathrm{kHz}$. 


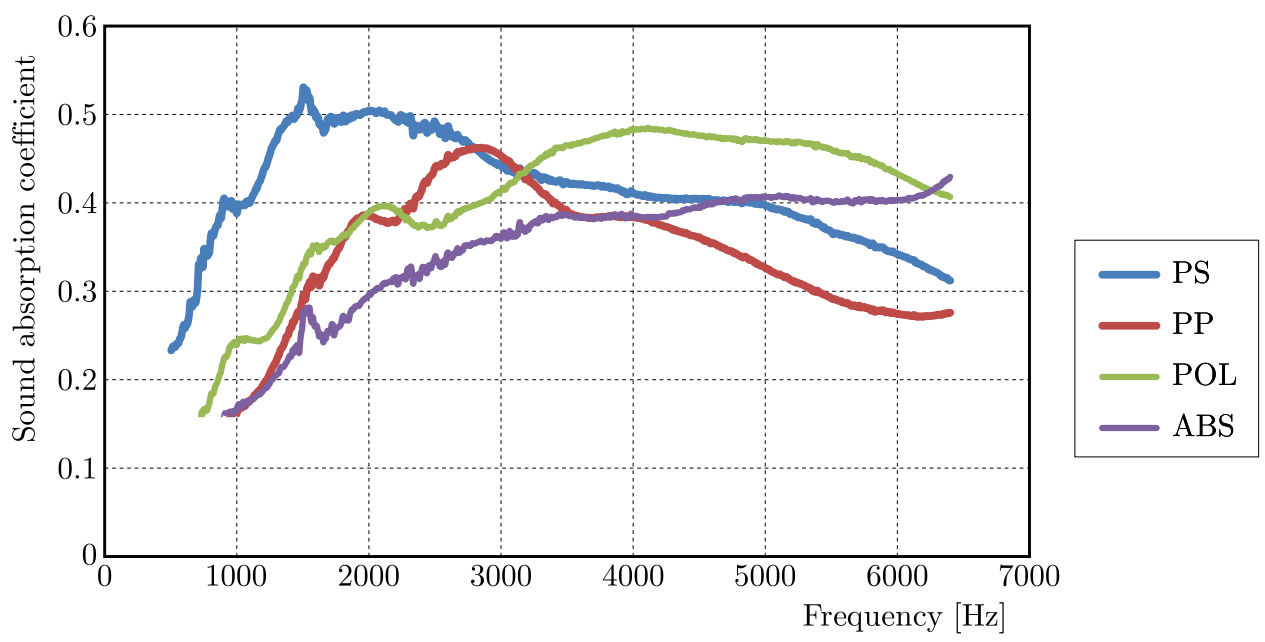

Fig. 8. Sound absorption coefficient for various granular materials subjected to the atmospheric pressure; sample II, front material type 2

\subsection{Influence of the sample length}

Figures 9-12 reveal the influence of length of the sample on the investigated acoustic properties. It is worth mentioning that many similarities between the compared characteristics can be encountered, e.g. the characteristic frequencies of resonance in the absorption coefficient spectra for corresponding specimens. The characteristics with front material type 1 (the thinnest) show more clearly that the shape of curves for sample I is similar for all materials which, thus, proves the impact of the sample front material is the least with type 1. With sample II, the results are also close for all grain types (there is a little variation with ABS).

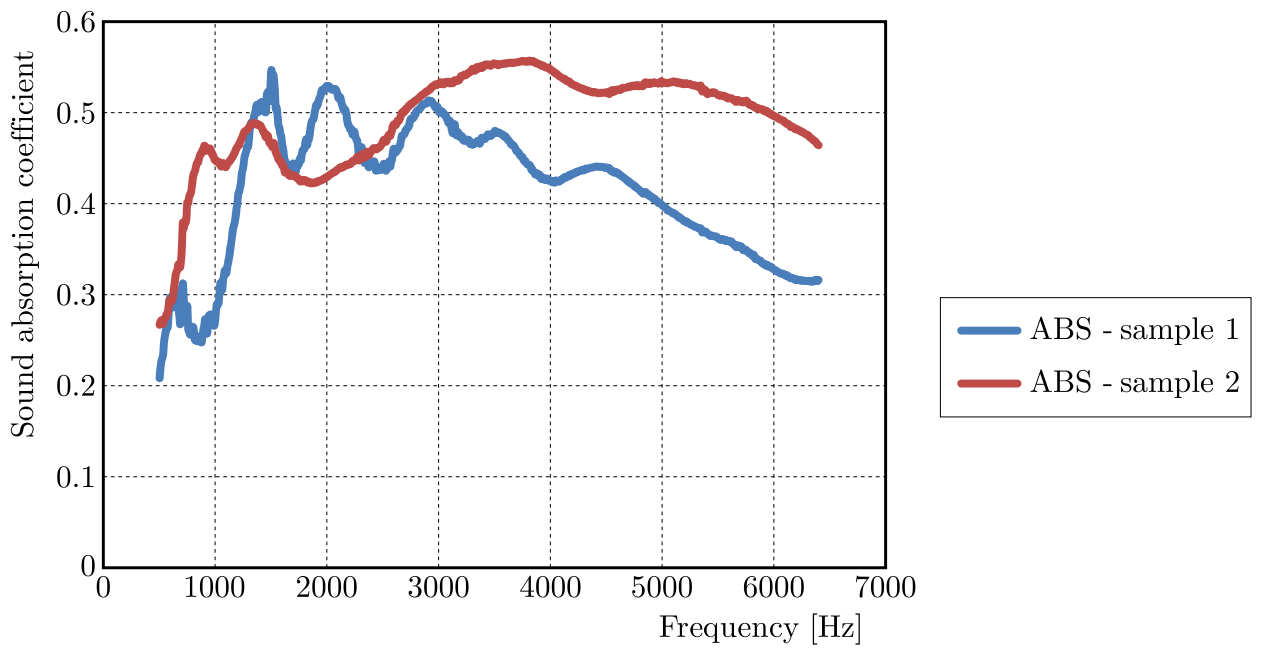

Fig. 9. Sound absorption coefficient for ABS grains; samples I and II, atmospheric pressure, front material type 1

The absorption coefficient is generally higher for sample II in comparison to sample I, which is particularly observable for frequencies above $3 \mathrm{kHz}$. Below that value, it depends on the sound frequency, since there are some regions with analogous properties for both specimens $(500 \mathrm{~Hz}-2 \mathrm{kHz})$. It would mean that the sample length is not a decisive factor for this range of frequencies. On the other hand, sample II in most cases (besides ABS) provides a more steady constant performance throughout the whole tested range. The results for sample I show a peak of performance at about $1.5 \mathrm{kHz}$, then the absorption is decreasing, also the shape of curves is wavy (sinusoidal), which is interesting, as it is almost a negligible feature for sample II. 


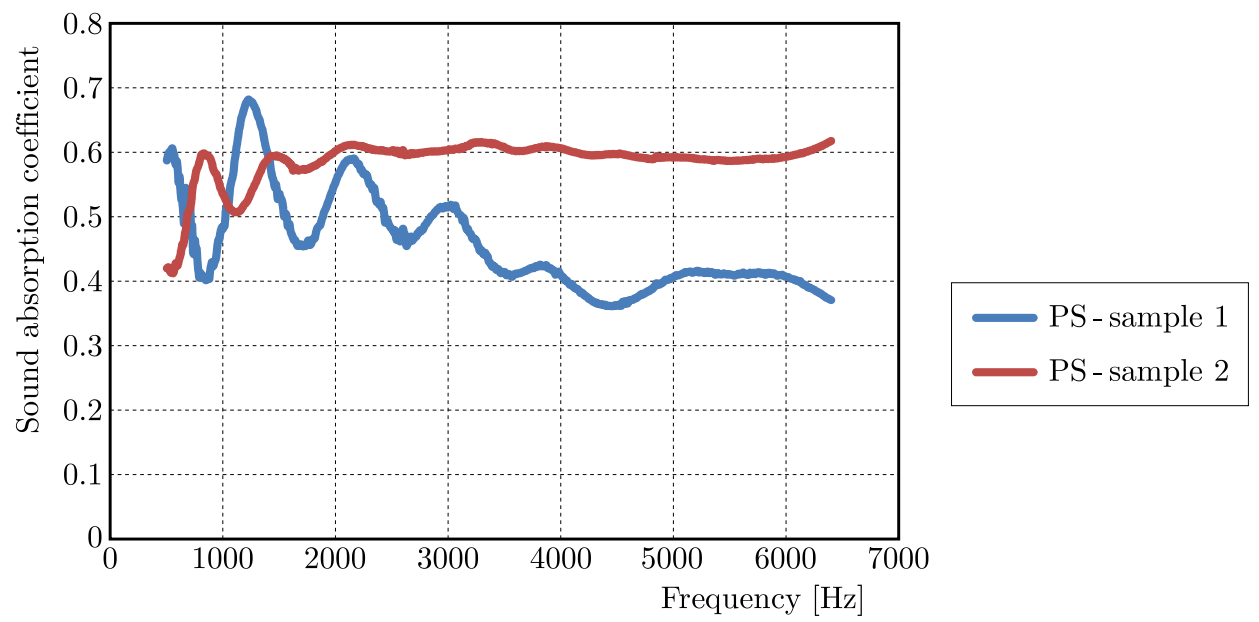

Fig. 10. Sound absorption coefficient for PS grains; samples I and II, atmospheric pressure, front material type 1

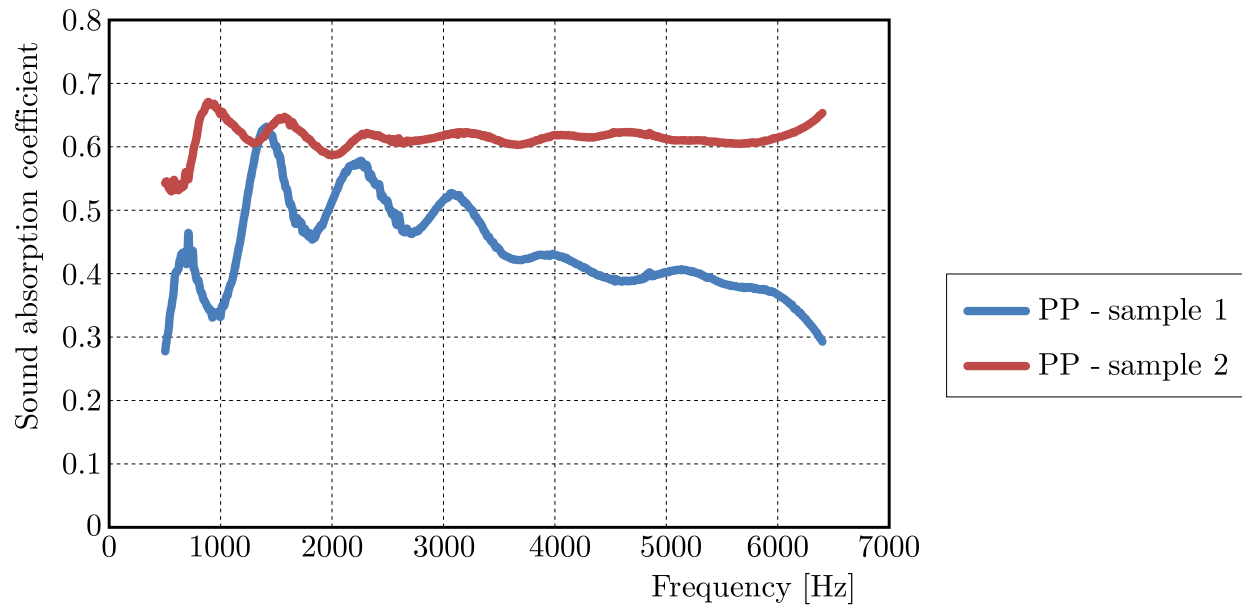

Fig. 11. Sound absorption coefficient for PP grains; samples I and II, atmospheric pressure, front material type 1

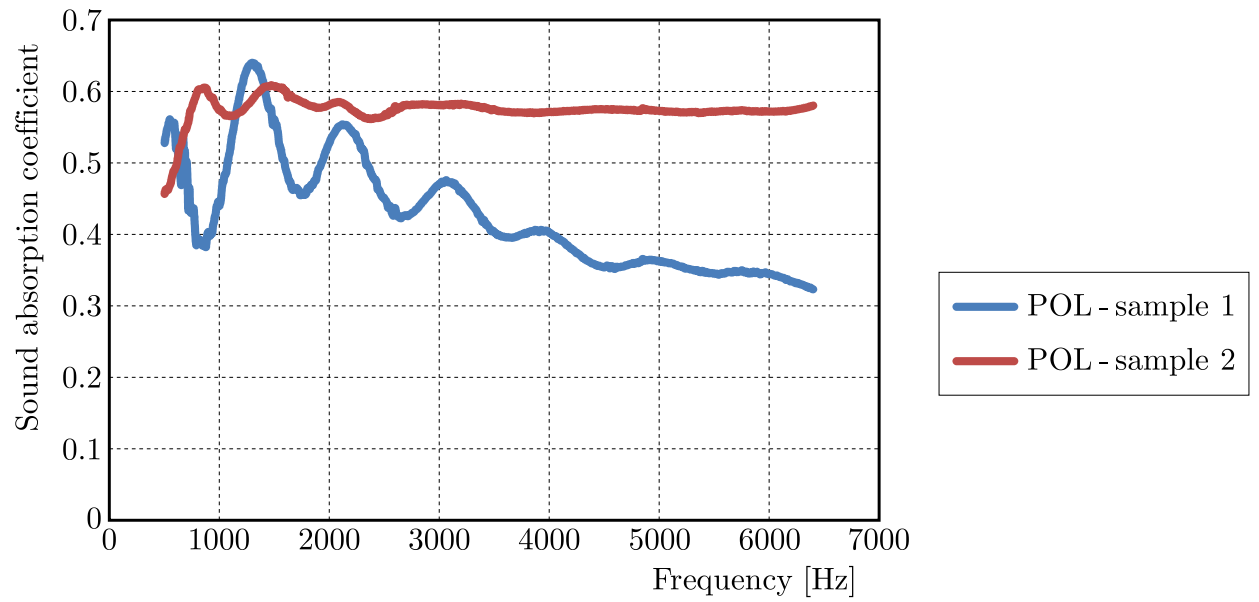

Fig. 12. Sound absorption coefficient for POL grains; samples I and II, atmospheric pressure, front material type 1 
It should be pointed out that the recorded characteristics for ABS grains reveal a range between 1.1 and $3 \mathrm{kHz}$, where the absorption level of sample I is greater than for sample II. Another thing to emphasize is that all the tested materials are of the same kind (plastics). And although there are physical differences between them (slight size, shape, properties etc.), the overall test results remain, in some ways, similar.

\section{4. $\quad$ Modeling}

To model the acoustic behavior of VPP, several models have been considered. As it turned out, the best suited is the one being developed by Voronina, and finally presented e.g. in (Voronina and Horoshenkov, 2004). The model involves a number of material parameters such as the characteristic dimension of particles $D$, tortuosity $q$ and porosity $H$ (being a ratio of the void space volume to the whole volume of the material). The model equations are

$$
\alpha_{0}=1-\left|\frac{z_{s}-1}{z_{s}+1}\right|^{2} \quad z_{s}=W \operatorname{coth}(\gamma l)
$$

where $\alpha_{0}$ is the absorption coefficient, $W=W_{a}+\mathrm{i} W_{i}$ is the normalized surface impedance, $\gamma=\alpha+\mathrm{i} \beta$ is the complex propagation constant, $z_{s}$ is the normalized surface impedance and $l$ is the thickness of the layer.

Also

$$
\alpha=\frac{k Q H}{1+A} \quad \beta=k[1+Q H(1+B)]
$$

and

$$
W_{i}=\frac{Q H}{1+C} \quad W_{a}= \begin{cases}1+Q & \text { for } \quad f<f_{c r} \\ \frac{q}{H} & \text { for } \quad F \geqslant f_{c r}\end{cases}
$$

where $k$ is the wavenumber in the air, $Q$ is the structural characteristic (acoustic parameter), $A, B$ and $C$ are certain coefficients, and $f_{c r}$ is the critical frequency.

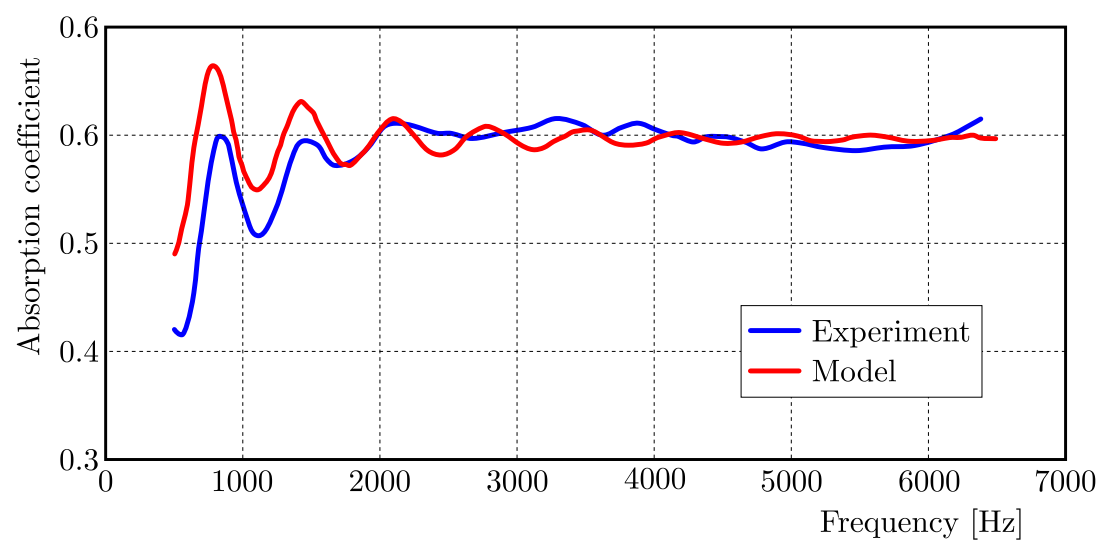

Fig. 13. Verification of experimental and numerical data for PS grains; sample II, atmospheric pressure, front material type 1

The first attempt of using the model for capturing the real acoustic behavior of VPP samples may be found in Fig. 13. It is clear that for normal conditions, the Voronina model works very well. The agreement between the model and experiment is satisfactory. Unlike other popular acoustic models (Biot, Allard, Delany-Bazley, etc.) proposed for various types of acoustic materials such as fibers or foams, the Voronina empirical formulation was developed especially for 
granular media. Basing on the data depicted in Fig. 13, it can be noticed that the calculated values of the absorption coefficient are close to the direct experimental results recorded in laboratory tests. Moreover, the character of both numerical and experimental characteristics is similar. It is worth mentioning that the modeled and recorded frequencies of resonance (e.g. $0.8 \mathrm{kHz}, 1,4 \mathrm{kHz}$ ) are coincident. Such observations confirm the correctness and reliability of the adopted Voronina model for capturing nonlinear acoustic properties of vacuum packed particles.

The Voronina model in presented form (4.1)-(4.3) does not include the underpressure parameter. To introduce nonlinear underpressure functions to the investigated model, additional laboratory tests have been carried out. The parameter of tortuosity was modified to compensate for the structural changes while introducing underpressure. It was observed that the mentioned changes did not include overall sample volume but, nevertheless, the grains were forced to closer contact and they could no longer freely vibrate, hence the tortuosity change. Two additional parameters alfa and beta (both as a function of $p$ ) were introduced to the tortuosity equation

$$
q=q_{0}+\left(\frac{f}{\alpha}\right)^{\beta}
$$

Two Figs. 14 and 15 show both parameters as a function of underpressure for two types of grains.

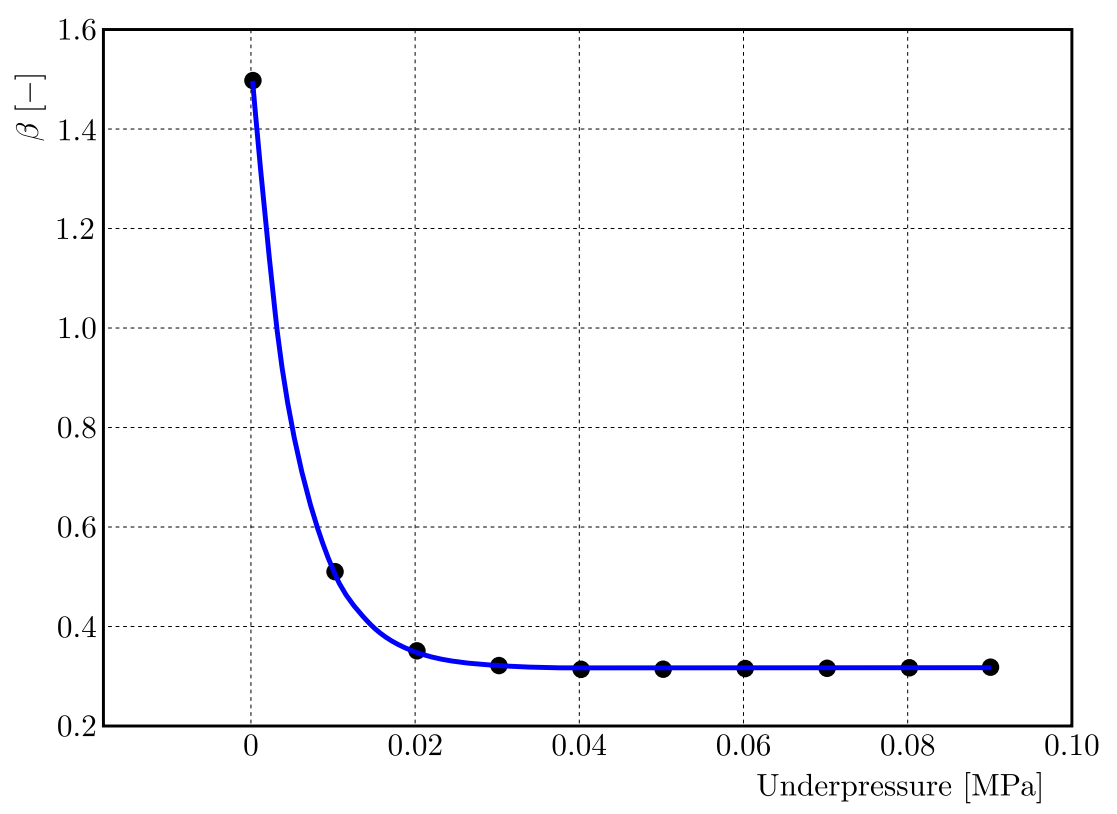

Fig. 14. Parameter $\beta$ as a function of underpressure for ABS grains

Subsequent Figs. 16-18 show the modeling with the use of new expressions against the experimental results.

\section{Conclusions and perspectives}

The current work is a continuation of previous efforts aimed at commercialization of Vacuum Packed Particles. The former papers of the authors were mainly devoted to studies and modeling of mechanical properties of VPP. It was shown in (Zalewski and Szmidt, 2014) that the Young modulus and proof stress (Szmidt, Zalewski, 2014) of such structures are complex functions of underpressure. In (Zalewski, 2013) the authors revealed that the volume of the specimen had an impact on the physical properties of granular conglomerates. Extraordinary features of VPP 


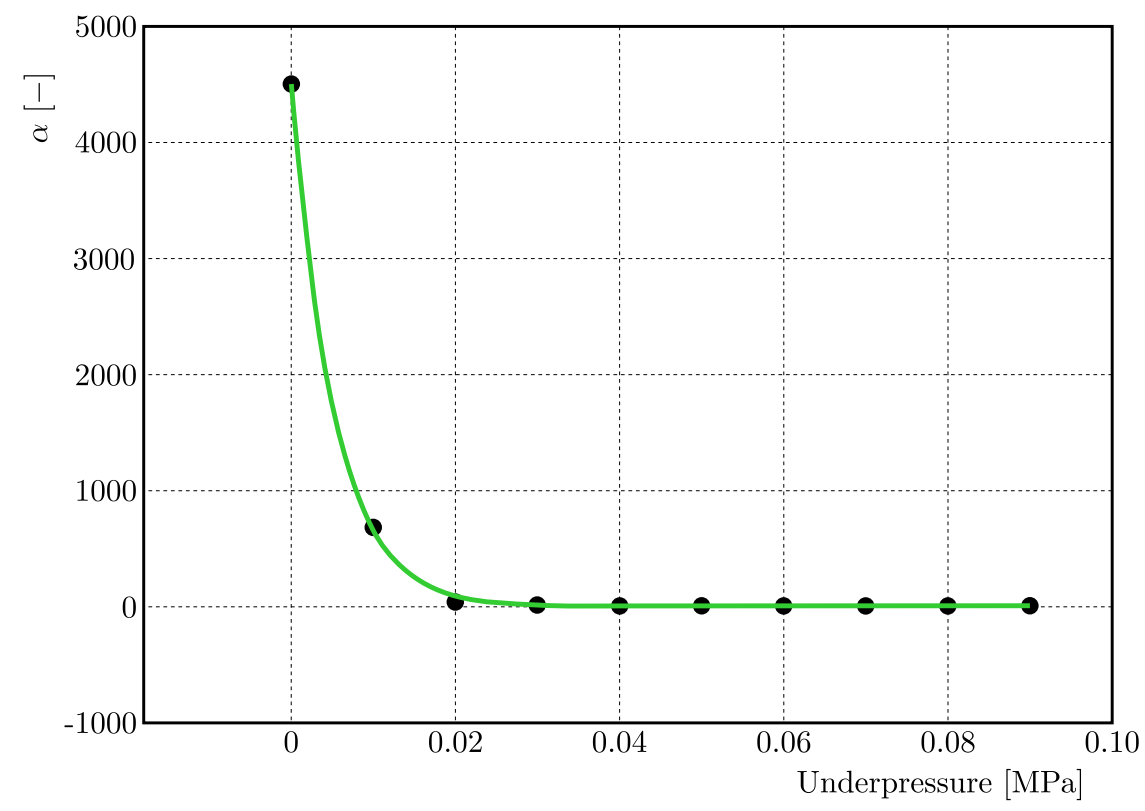

Fig. 15. Parameter $\alpha$ as a function of underpressure for PMMA grains

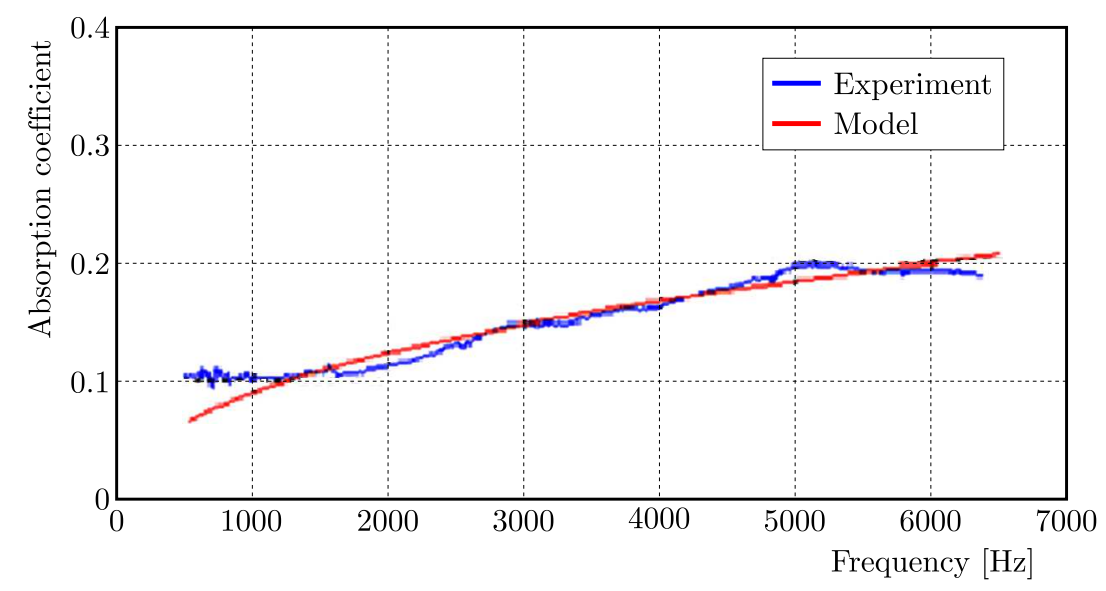

Fig. 16. Verification of experimental and numerical data for PMMA grains; sample I, underpressure $0.01 \mathrm{MPa}$, front material type 2

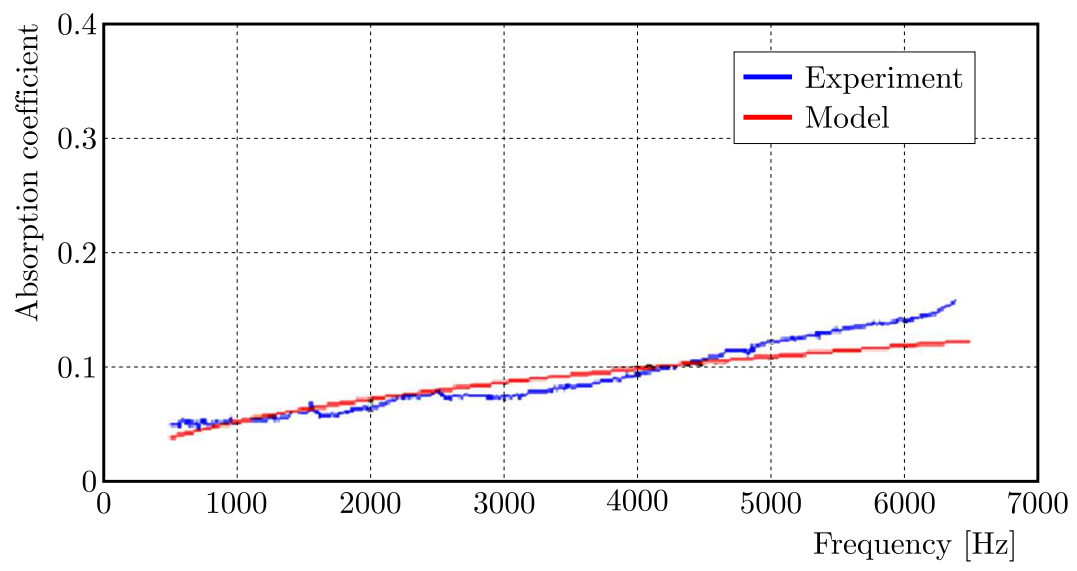

Fig. 17. Verification of experimental and numerical data for ABS grains; sample II, underpressure $0.01 \mathrm{MPa}$, front material type 2 


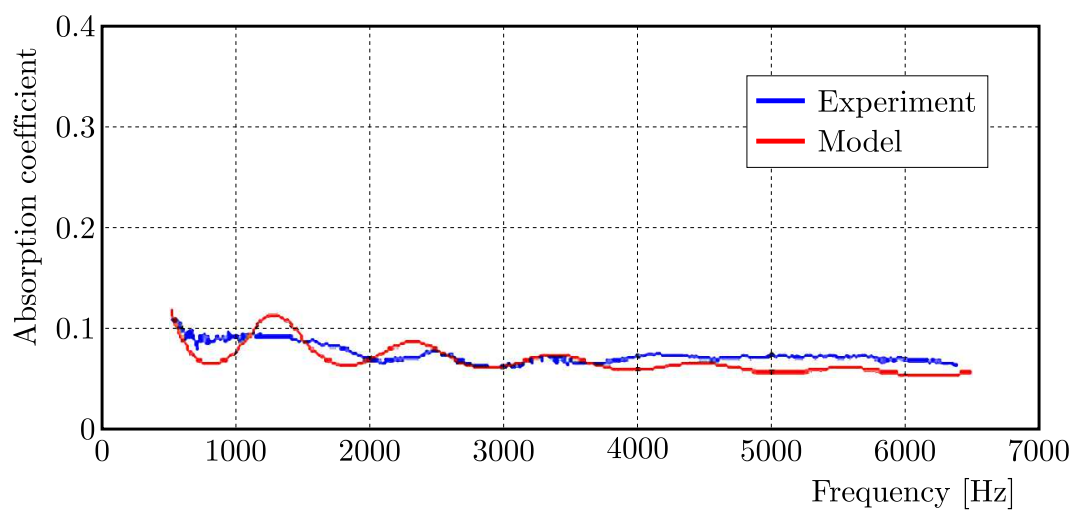

Fig. 18. Verification of experimental and numerical data for PMMA grains; sample I, underpressure $0.09 \mathrm{MPa}$, front material type 1

observed in the former experiments encouraged the authors to focus on the acoustic properties of VPP.

Studying sound absorbing characteristics of Vacuum Packed Particles has confirmed to be a very interesting and far-reaching task. It revealed that the simple mechanism of vacuum packing and changing the internal pressure can widely influence the acoustic characteristics of tested materials. Although there are some obvious limitations in practical engineering applications of VPP resulting from incomplete knowledge about their behavior, the control possibilities alone make the authors believe that the future work will help them to overcome issues that have been observed during the current research.

Moreover, using VPP as a part of an active sound absorber is possible but requires further work aiming at maximizing the sample absorption in a loose state of the granular mix while minimizing its thickness.

The presented results clearly depict that there is a simple way of influencing the structure and properties of VPP. Having performed several hundred measurements, the authors can clearly state that while materials selected for the test may seem similar in a physical way, the detailed comparison of complete results reveals many incoherences in their absorption characteristics (e.g. Figs. 8 or 9). It is clear that even small differences in the shape of the grains or in other micro-features will have a great impact on the macroscopic physical (acoustic) properties of VPP.

Nevertheless, there are still many aspects to be considered in further research. Open questions include among others the influence of the mentioned physical factors (dimension, shape) or the compression ratio of grains on the acoustic properties. These parameters seem to be particularly significant because they undoubtedly determine such parameters as porosity or tortuosity (Allard, 1993; Attenborough, 1983) of tested specimens.

Another interesting issue is the quantitative change of the absorption coefficient in the restricted range of internal underpressures form 0 to $0.01 \mathrm{MPa}$. In the perspective research, this range of partial vacuum will be closely examined to record and describe the nature of the mentioned changes. In further research, it will be also important to consider materials of different strength properties (Karliński et al., 2016). Grains would probably demonstrate alternative behavior when made of rubber or another deformable elastic material.

\section{References}

1. Allard J.F., 1993, Propagation of Sound in Porous Media. Modelling Sound Absorbing Materials, Elsevier, Amsterdam 
2. Attenborough K., 1983, Acoustical characteristics of rigid fibrous absorbents and granular materials, Journal of the Acoustical Society of America, 73, 785-799

3. Bajkowski J., Jasiński M., Mączak J., Radkowski S., Zalewski R., 2012, The active magnetorheological support as an element of damping of vibrations transferred from the ground to large-scale structure supports, Key Engineering Materials, 518, 350-357

4. Bajkowski J.M., Zalewski R., 2014, Transient response analysis of a steel beam with vacuum packed particles, Mechanics Research Communications, 60, 1-6

5. Besset S., IchChou M.N., 2011, Acoustic absorption material optimisation in the mid-high frequency range, Applied Acoustics, 72, 632-638

6. Brown E., Rodenberg N., Amend J., Mozeika A., Steltz E., Zakin M.R., Lipson H., JAEger H.M., 2010, Universal robotic gripper based on the jamming of granular material, Proceedings of the National Academy of Sciences of the United States of America, 107, 18809-18814

7. ERsoy S., KüÇÜK H., 2009, Investigation of industrial tea-leaf-fibre waste material for its sound absorption properties, Applied Acoustics, 70, 1, 215-220

8. Fatima S., Mohanty A.R., 2011, Acoustical and fire-retardant properties of jute composite materials, Applied Acoustics, 72, 2/3, 108-114

9. Gawdzińska K., Chybowski L., Bejger A., Krile S., 2016, Determination of technological parameters of saturated composites based on $\mathrm{SiC}$ by means of a model liquid, Metalurgija, 55, 4, 659-662

10. Gawdzińska K., Chybowski L., Przetakiewicz W., 2015, Proper matrix-reinforcement bonding in cast metal matrix composites as a factor of their good quality, Archives of Civil and Mechanical Engineering, 16, 3, 553-563

11. Hong Z., Bo L., Guangsu H., JiA H., 2007, A novel composite sound absorber with recycled rubber particles, Journal of Sound and Vibration, 304, 1/2, 400-406

12. ISO 10534-2, Acoustics - Determination of sound absorption coefficient and impedance in impedance tubes - Part 2: Transfer-function method

13. Karliński J., Ptak M., Dziąak P., Rusiński E., 2014, Strength analysis of bus superstructure according to Regulation No. 66 of UN/ECE, Archives of Civil and Mechanical Engineering, 14, 342-353

14. Karliński J., Ptak M., DziaŁak P., Rusiński E., 2016, The approach to mining safety improvement: accident analysis of an underground machine operator, Archives of Civil and Mechanical Engineering, 16, 3, 503-512

15. Pyrz M., ZaleWski R,, 2010, Modeling of granular media submitted to internal underpressure, Mechanics Research Communications, 37, 2, 141-144

16. Szmidt T., Zalewski R., 2014, Inertially excited beam vibrations damped by Vacuum Packed Particles, Smart Materials and Structures, 23, 2014, 105026 (9 pp)

17. Swift M.J., Briš P., Horoshenkov K.V., 1999, Acoustic absorption in re-cycled rubber granulate, Applied Acoustics, 57, 203-212

18. Voronina N.N., Horoshenkov K.V,, 2003, A new empirical model for the acoustic properties of loose granular media, Applied Acoustics, 64, 415-432

19. Voronina N.N., Horoshenkov K.V., 2004, Acoustic properties of unconsolidated granular mixes, Applied Acoustics, 64, 673-691

20. Wilson D.K., 1997, Simple, relaxational models for the acoustical properties of porous media, Applied Acoustics, 50, 3, 171-188

21. Yamamoto T., Maruyama S., Nishiwaki S., Yoshimura M,, 2009, Topology design of multi-material soundproof structures including poroelastic media to minimize sound pressure levels, Computer Methods in Applied Mechanics and Engineering, 1439-1455 
22. Zalewski R., 2010, Constitutive model for special granular structures, International Journal of Non-Linear Mechanics, 45, 3, 279-285

23. Zalewski R., 2013, Modeling and Research of the Underpressure Influence on Mechanical Properties of Vacuum Packed Particles (in Polish), WKŁ, Warsaw, ISBN 978-83-206-1851-8

24. Zalewski R., Nachman J., Shillor M., Bajkowski J., 2014, Dynamic model for a magnetorheological damper, Applied Mathematical Modelling, 38, 9/10, 2366-2376

25. Zalewski R., Szmidt T., 2014, Application of Special Granular Structures for semi-active damping of lateral beam vibrations, Engineering Structures, 65, 13-20

26. Zieliński T.G., 2011, Numerical investigation of active porous composites with enhanced acoustic absorption, Journal of Sound and Vibration, 5292-5308

Manuscript received December 11, 2017; accepted for print January 26, 2018 